\title{
Üçgensel Müdahaleli Yarı-Markov (s,S) Tipli Modellerin Momentleri İçin Tahmin Ediciler
}

\author{
Estimators of the Moments for a Semi-Markovian Model of Type $(s, S)$ With a Triangular \\ Distributed Interference of Chance
}

\author{
Melek ERİȘ BÜYÜKKAYA ${ }^{1, a}$, Aslı BEKTAŞ KAMIȘLIK ${ }^{2, b}$, Tülay KESEMEN*3,c \\ ${ }^{1}$ Karadeniz Teknik Üniversitesi, Fen Fakültesi, İstatistik ve Bilgisayar Bilimleri Bölümü, 61080 Trabzon \\ ${ }^{2}$ Recep Tayyip Erdoğan Üniversitesi, Fen Edebiyat Fakültesi, Matematik Bölümü, 53100, Rize \\ ${ }^{3}$ Karadeniz Teknik Üniversitesi, Fen Fakültesi, Matematik Bölümü, 61080, Trabzon
}

• Geliş tarihi / Received: 20.02.2018 • • Düzeltilerek geliş tarihi / Received in revised form: 09.05.2018 • Kabul tarihi / Accepted: 15.05.2018

\begin{abstract}
$\ddot{O} z$
$\mathrm{Bu}$ çalı̧̧mada üçgensel müdahaleli $(\mathrm{s}, \mathrm{S})$ tipli yarı-Markov bir envanter(stok kontrol) model yenileme süreci ele alınmış, ve bu sürecin ergodik dağılımının momentleri için tahmin edici problemi araştırılmıştır. Yenileme süreçlerinde, yenilemeler arasında geçen sürenin dağılımı tam olarak bilinmediğinde yenileme fonksiyonu için tahmin ediciler literatürde mevcuttur. Bu çalışmanın ana motivasyonu ise, Frees (1986b) tarafindan yenileme fonksiyonu için önerilmiş tahmin edicidir. Bu amaçla Frees (1986b)'in yaklaşımı kullanılarak ele alınan modelin ergodik dağılımının momentleri için istatistiksel bir tahmin edici bulunmuş ve bu tahmin edici için sırasıyla tutarlılık, asimptotik yansızlık ve asimptotik normallik gibi istatistiksel özellikler araştırılmıştır.
\end{abstract}

Anahtar kelimeler: $(s, S)$ tipli yarl-Markov envanter model, Asimptotik Normallik, Asimptotik Yansızlık, Istatistiksel tahmin edici, Tutarlılık

\begin{abstract}
In this study inventory model of type $(s, S)$ with triangular interference of chance has been investigated by using renewal process and the statistical estimator problem for moments of ergodic distribution of the process which define the model has been obtained. Different estimators for the renewal function are available in the literature, when the distribution which defines the time between renewals is not known precisely. The main motivation of this study is the publication by Frees (1986b) in which he proposed a statistical estimator for the renewal function. By using the estimator by Frees (1986b) we obtained a new estimator for the moments of ergodic distribution function of the process describing inventory model of type $(s, S)$. Moreover statistical properties such as consistency, asymptotic unbiasedness and asymptotic normality were investigated for the obtained estimator, respectively.
\end{abstract}

Keywords: Inventory model of type $(s, S)$, Asymptotic Normality, Asymptotic Unbiasedness, Statistical estimators, Consistency

\footnotetext{
*c Tülay KESEMEN; tkesemen@gmail.com; Tel: (0462) 37725 70; orcid.org/0000-0002-8807-5677

${ }^{a}$ orcid.org/0000-0002-6207-5687 $\quad{ }^{b}$ orcid.org/0000-0002-9776-2145
} 


\section{Giriş}

$(\mathrm{s}, \mathrm{S})$ tipli yar1-Markov envanter modeller stok kontrol, kuyruk teorisi, stokastik finans gibi aktüeryanın önemli problemlerinde yaygın olarak karşılaşılan süreçlerdir. Dolayısı ile $(\mathrm{s}, \mathrm{S})$ tipli yar1-Markov envanter modellerin sayisal ve istatistiki karakteristiklerini incelemek envanter teorisi ile ilgili problemlerin çözümünde oldukça önemli bir role sahiptir. Literatürde bu modellerin asimptotik olarak incelenmesini ele alan pek çok çalışma mevcuttur (Kesemen vd., 2016; Khaniyev ve Atalay, 2010; Khaniyev vd., 2013).

Yar1-Markov envanter modelini ifade eden sürecin ergodik dağılımı ve ergodik dağılımının momentleri bazı durumlar dışında kolaylıkla elde edilememektedir. Böyle durumlarda kesikli müdahaleli yar1-Markov envanter modeller yenileme fonksiyonları $\operatorname{arac1lığ} 1$ ile ifade edilebilir. Yenileme teorisine bir elektrik ampul sigortası veya sınırlı bir ömre sahip diğer bir ekipman parçası standart örnek olarak verilebilir. Parça bozulur bozulmaz yeni parça ile değiştirilir ve süreç bu şekilde devam eder (Feller, 1971). Yenileme teorisinde genel olarak bu parçaların arızasız biçimde çalıştığı süreleri temsil eden bağımsız aynı dağılıma sahip $\left(X_{i}\right)_{i \in \mathbb{N}}$ rasgele değişkenleri bulunur ve bu rasgele değişkenlerin k1smi toplamları, $S_{0}=0, S_{n}=\sum_{i=1}^{n} X_{i}$ olarak gösterilir. Bu durumda $N(t)=\sup \left\{n \in \mathbb{N}_{0}: S_{n} \leq\right.$ $t$ \} biçiminde tanımlanan süreç " $t$ " anına kadar meydana gelen yenilemelerin sayısıdır. Bu tip bir yap1 genellikle [s,S] tipli yarı- Markov envanter modeller de dahil olmak üzere pek çok karmaşık stokastik yapının modellenmesinde kullanılır. Yenileme teorisi, bu tür modellerin analizi için bazı temel teknikler sağlar ve yenileme fonksiyonu $U(t)=E(N(t))$ bu modellerin analizinde anahtar rolü oynar. $\mathrm{Bu}$ nedenle yenileme fonksiyonunun asimptotik açılımını ya da yenileme fonksiyonu için elde edilmiş olan tahmin edicileri bilmek, (s,S) tipli stok kontrol modelleri de dahil olmak üzere, yenileme süreçleri ile ifade edilen modellerin karakteristiklerinin elde edilmesi açısından oldukça önemlidir. Fakat yenilemeler arası geçen ardışık zaman sürelerinin dağılımı bilinmiyorken, yenileme fonksiyonu olan $U(t)^{\prime}$ nin kesin ifadesine ulaşmak her zaman mümkün olmayabilir. Böyle durumlarda yenileme fonksiyonu verilerden tahmin edilmek durumundadır. $U(t)$ yenileme fonksiyonu, yenileme teorisi yardımı ile ifade edilen pek çok uygulamada rol oynadığından, yenileme fonksiyonunun istatistiki tahmin edicileri ile ilgili çalışmalar günümüzde önemli bir araştırma alanı haline gelmiştir.

Yenileme fonksiyonunun tahmin edilmesi ile ilgili problemler literatürde bazı yöntemlerle ele alınmıştır. Vardi (1982) yenileme fonksiyonunun parametrik olmayan en çok olabilirlik tahmin edicisini bir algoritma kullanarak elde etmiştir. Feller (1971) yenileme fonksiyonunun iki terimli asimptotik açılımını

$$
U(t)=\frac{t}{\mu_{1}}+\frac{\mu_{2}}{2 \mu_{1}^{2}}+o(1)
$$

olarak ifade etmiştir. t' nin yeterince büyük değerleri için Feller (1971)' in önerdiği asimptotik ifadeye bağl1 tahmin edicisi parametrik olmayan bir yaklaşım kullanılarak Frees (1986 b) tarafından aşağıdaki gibi önerilmiştir:

$$
\widehat{U}(t)=\frac{t}{\hat{\mu}_{1}}+\frac{\hat{\mu}_{2}}{2 \hat{\mu}_{1}^{2}}
$$

Frees (1986 b) çalışmasında elde ettiği tahmin edicinin küçük $\mathrm{t}$ değerleri için tutarlı olmadığını ispatlamış fakat büyük $t$ değerleri için bu tahmin edicinin istatistiki özelliklerini araştırmamıştır. $\mathrm{Bu}$ tahmin edicinin istatistiki özellikleri ilk defa Gökpınar (2016b), Gökpınar vd. (2015a, 2015b), Gökpınar vd. (2016a) ve Gökpınar vd. (2018) çalışmalarında ele alınmıştır. Frees (1986 a) aynı zamanlarda yaptığı başka bir çalışmada yenileme fonksiyonu için hem $\mathrm{U}$ hem de $\mathrm{V}$ istatistiğine dayalı parametrik olmayan tahmin ediciler önermiştir. Schneider vd. (1990) yaptıkları çalışmada yenileme fonksiyonu için parametrik olmayan bir tahmin edici önermişlerdir ve bu tahmin ediciyi Frees'in önerdiği tahmin ediciler ile karşılaştırmışlardır. Grübel ve Pitts (1993), Schneider vd. (1990) ve Frees (1986 a) tarafindan yenileme fonksiyonu için tek taraflı olarak elde edilmiş olan tahmin edici problemini iki taraflı duruma taşımışlardır. Frees (1986 b) tahmin edicisine benzer bir histogram tipli tahminci, Markovich ve Krieger (2006) tarafindan önerilmiştir. $E\left(X^{2}\right)=\infty$ durumu için Sgibnev (1981), $U(t)$ yenileme fonksiyonu için aşağıdaki asimptotik sonucu elde etmiştir:

$$
U(t)-\frac{t}{\mu_{1}} \sim \frac{1}{\mu_{1}^{2}} \int_{0}^{t}\left(\int_{y}^{\infty} \bar{F}(x) d x\right) d y .
$$


Burada $\bar{F}=1-F, F$ dağılımının kuyruğudur. $F$ ise $U(t)$ yenileme fonksiyonunu üreten rasgele değişkenlere ait dağılım fonksiyonudur. $\mathrm{Bu}$ asimptotik sonuç kullanılarak $E\left(X^{2}\right)=\infty$ durumunda Bebbington (2007) tarafindan $U(t)$ yenileme fonksiyonunun parametrik olmayan tahmin edicisi

$$
U_{n}(t)=\frac{t}{\hat{\mu}_{1}}+\frac{1}{\hat{\mu}_{1}^{2}} \int_{0}^{t}\left(\int_{y}^{\infty} \overline{F_{n}}(x) d x\right) d y
$$

biçiminde elde edilmiştir. Burada $\hat{\mu}_{1}, \quad F$ dağılımının birinci örneklem ortalamasını temsil eder. Bebbington (2007) tarafindan elde edilen bu sonuç, $F$ dağılım fonksiyonu $\frac{1}{2}<\alpha<1$ indeksi ile $S_{\alpha}$ nın kararlılık çekim alanında olduğunda $U(t)$ için güven aralıklarının elde edilmesine de olanak sağlamıştır. Daha sonra Necir vd. (2010) tarafından yenilemeler arası geçen zaman ağır kuyruklu bir dağılıma sahip iken uç değer teorisi ve ESAD yöntemi kullanılarak yenileme fonksiyonu için Bebbington tarafından önerilmiş olan tahmin edicinin daha kullanışlı bir formu elde edilebilmiştir.

Yenileme sürecinde yenilemeler arasında geçen sürenin dağılımı $\mathrm{F}$ bilinmediğinde yenileme fonksiyonunun tahmini için, yukarıda da belirtildiği gibi birçok tahmin edici önerilmiştir. Fakat bu tahmin edicilerin birçoğunu pratik hayata uygulamak, özellikle büyük $t$ değerleri için oldukça zordur. Fress (1986 b) büyük t değerleri için örnek momentlere dayanan bir tahmin edici önermiştir. $\mathrm{Bu}$ tahmin ediciyi uygulamak zor değildir. $\mathrm{Bu}$ nedenle, bu tahmin edicinin asimptotik yansız, tutarlılık ve normallik gibi bazı istatistiksel özellikleri araştııılmış ve farklı dağılımlar altında bu tahmin edicinin performansı simülasyon çalışmalarında incelenmiştir (Gökpınar, 2016b). Elde edilen sonuçlar, bu tahmin edicinin farklı $t$ ve $n$ değerlerine göre yenileme fonksiyonu $U(t)$ ye oldukça yakın değerler verdiğini göstermiştir. $(\mathrm{s}, \mathrm{S})$ tipli stok kontrol modellerinin uzun süredeki davranış karakteristiklerini (dağılım fonksiyonu, momentleri, vb.) bilmek önemli olduğu için bu tahmin edicinin kullanılması yenileme fonksiyonu içeren karmaşı stok kontrol modelleri için gereklidir. Fakat günlük hayatta talep değişkenine ait dağılım fonksiyonu $\mathrm{F}$ genelde bilinmediğinden, $(\mathrm{s}, \mathrm{S})$ tipli stok kontrol modellerinin uzun süredeki davranış karakteristiklerini tahmin etmek gerekmektedir. $\mathrm{Bu}$ amaçla, bu çalışmada Frees (1986 b)' in önerdiği tahmin edici kullanılarak, $(\mathrm{s}, \mathrm{S})$ tipli stok kontrol modelini ifade eden sürecin ergodik dağılımının momentleri için tahmin edici bulunmuş ve elde edilen tahmin edicinin sırasıyla tutarlılık, asimptotik yansızlık ve asimptotik normallik gibi istatistiksel özellikler araştırılmıştır.

\section{1. (s,S) Tipli Stok Kontrol Modeli}

$\mathrm{Bu}$ bölümde, (s,S) tipli envanter (stok kontrol) modeli tanıtılmıştır. Buna göre bu modelin matematiksel tanımı verilmeden önce temel notasyonlar aşağıdaki gibidir.

$Y(t)$ : t anında bir depodaki stok seviyesi,

$\eta_{n}$ : Talep miktarlarını temsil eden rasgele değişkenler,

$\xi_{n}$ : Talepler arasında geçen süreyi temsil eden rasgele değişkenler,

$\tau_{1}$ : Stok seviyesinin $\mathrm{s}$ seviyesinin altına düştüğü ilk an,

$\zeta_{n}$ : Kesikli müdahaleyi ifade eden rasgele değişken,

$N_{1}$ : Stok seviyesinin $\mathrm{s}$ seviyesinin altına düştügü ana kadar gerekli talep sayısı,

s: $\quad$ Stok kontrol seviyesi,

$S: \quad$ Maksimum stok seviyesi,

Depoda başlangıç $t=0$ anındaki stok seviyesi $Y(0) \equiv Y_{0} \equiv z \in[s, S) \quad$ olsun. Depodaki stok seviyesinin önceden belirlenmiş bir s seviyesinin altına düştügü ana kadar her bir rasgele $\left(T_{n}\right)$ anında sisteme rasgele $\left(\eta_{n}\right)$ miktarında talepler gelmektedir. Bu durumda

$$
T_{n}=\sum_{i=1}^{n} \xi_{i}
$$

biçiminde olacaktır. Buna göre depodaki stok seviyesi değişimi aşağıdaki gibi olur:

$Y\left(T_{1}\right) \equiv Y_{1}=z-\eta_{1}$
$Y\left(T_{2}\right) \equiv Y_{2}=z-\left(\eta_{1}+\eta_{2}\right)$
$\vdots$
$Y\left(T_{n}\right) \equiv Y_{n}=z-\sum_{i=1}^{n} \eta_{i}$.

Görüldüğü gibi depodaki stok miktarının değişimi $\left\{\eta_{i}\right\}, \quad i \geq 1$ doğrultusundadır. $\mathrm{Bu}$ değişim depodaki stok miktarı "s" stok kontrol seviyesinin altına ilk düştügü ana yani $\tau_{1}$ ' e kadar devam eder. Depodaki stok seviyesi önceden belirlenmiş bir s seviyesinin altına düştüğü anda $\zeta_{1}$ seviyesine kadar stokla doldurulur. Böylece ilk periyot tamamlanmış olur. İkinci periyot başlangıç 
seviyesi olarak $\zeta_{1}$ seviyesinden başlar ve ilk periyoda benzer şekilde devam eder. $\mathrm{Bu}$ çalışmada ele aldığımız $(s, S)$ tipli envanter model (stok kontrol) belirli koşulları sağlamaktadır. Buna göre sistemde iki ardışık talep miktarı arasında geçen süreleri ifade eden $\left\{\xi_{i}\right\}, i \geq 1$ rasgele değişkenleri, talep miktarlarını ifade eden $\left\{\eta_{i}\right\}, i \geq 1$ rasgele değişkenleri ve müdahaleyi ifade eden $\left\{\zeta_{i}\right\}, i \geq 1$ rasgele değişkenleri bağımsız aynı dağılımlıdırlar. Bunlara ek olarak $\xi_{i}, \eta_{i}$ ve $\zeta_{i}$ rasgele değişkenleri de birbirinden bağımsızdır.

$\mathrm{Bu}$ çalışmada, $(\mathrm{s}, \mathrm{S})$ tipli envanter modelin ergodik dağılımının momentleri için tahmin edici bulma problemi ele alınmış ve bu tahmin edicilerin bazı istatistiksel özellikleri araştırılmıştır. Bu kapsamda yapılan çalışmalar kısaca şu şekilde olacaktır: Bölüm 2' de (s, S) tipli envanter modeli ifade eden sürecin matematiksel kurulumu verilmiştir. Bölüm 3' de bu sürecin momentleri için Frees (1986 b)' in tahmincisine dayanan tahmin edici önerilmiş, son olarak ise bu tahmin edici için bazı istatistiksel özellikler araştırılmıştır.

\section{Y(t) Sürecinin Matematiksel Kuruluşu}

$\left\{\xi_{n}, \eta_{n}, \zeta_{n}\right\}, n \geq 1 \quad$ ayn $1 \quad(\Omega, F, P) \quad$ olasıllk uzayında tanımlanmış bağımsız aynı dağılıma sahip rasgele değişkenler dizisi olsun. Burada $\xi_{n}$ rasgele değişkenleri keyfi dağılıma sahip olduğundan yar1- Markov özelliğini sağlamaktadır. Ayrıca $\xi_{n}$ ve $\eta_{n}$ rasgele değişkenleri pozitif değerler alırken, $\zeta_{n}$ rasgele değişkenleri $[s, S)$ aralı̆̆ında değerler alır. $\xi_{n}, \eta_{n}$ ve $\zeta_{n}$ rasgele değişkenleri kendi aralarında da bağımsız rasgele değişkenlerdir ve dağılım fonksiyonları sırası ile aşağıdaki gibidir:

$\Phi(t)=P\left\{\xi_{1} \leq t\right\}$,

$F(x)=P\left\{\eta_{1} \leq x\right\}$,

$\pi(z)=P\left\{\zeta_{1} \leq z\right\}, \pi(0)=0$.

$\xi_{n}, \quad \eta_{n}$ ve $\zeta_{n}$ başlangıç rasgele değişkenleri aracılığıyla, $T_{n}$ ve $Z_{n}$ yenileme dizileri aşağıdaki gibi tanımlansin:

$T_{n}=\sum_{i=1}^{n} \xi_{i}, \quad Z_{n}=\sum_{i=1}^{n} \eta_{i}, n \geq 1, \quad T_{0}=$ $Z_{0}=0$.

$\left\{N_{n}\right\}$ tam değerli rasgele değişken dizisi aşağıdaki gibi tanımlansın:

$N_{0}=0, N_{1}=\min \left\{k \geq 1: z-Z_{k}<s\right\}, N_{n+1}=$ $\min \left\{k \geq N_{n}+1: \zeta_{n}-\left(Z_{k}-Z_{N_{n}}\right)<s\right\}$
$N_{1},\left\{Z_{n}\right\}$ tarafından "s" seviyesini ilk geçiş zamanı olan $\tau_{1}$ anına kadar gerekli talep sayısıdır. Ayrica

$$
\begin{aligned}
\tau_{0}=0, \tau_{1}(z)= & T_{N_{1}}=\sum_{i=1}^{N_{1}} \xi_{i} ; \tau_{n}=T_{N_{n}} \\
& =\sum_{i=1}^{N_{n}} \xi_{i} ; n \geq 2
\end{aligned}
$$

olmak üzere $v(t)=\operatorname{maks}\left\{n \geq 0: T_{n} \leq t\right\}, t>0$ tanımlansin. $\mathrm{Bu}$ durumda, depodaki stok miktarının değiş̧imini gösteren $Y(t)$ süreci aşağıdaki gibi inşaa edilir:

$$
\begin{aligned}
& Y(t)=\zeta_{n}-\left(Z_{v(t)}-Z_{N_{n}}\right), \\
& \tau_{n} \leq t<\tau_{n+1} ; \quad n=0,1,2, \ldots .
\end{aligned}
$$

Burada $\zeta_{0}=z \in[s, S]$ dir.

\section{Y(t) Sürecinin Ergodik Dağılımının Momentleri}

Khaniyev vd. (2013) yaptı̆ğ çalışmasında $Y(t)$ ifadesi ile matematiksel kurulumu verilmiş olan sürecin ergodiklik koşulları ve bu koşullar altında genel ergodiklik teoreminin şartlarını sağladığını göstermiştir. Ayrıca Khaniyev vd. (2013) notasyon kolaylığ $\breve{1}_{1}$ açısından $Y(t)$ sürecinin ergodik dağılımının $n$. dereceden momentleri $E\left(Y^{n}\right)$ ile gösterilmiştir. Bu durumda $E\left(Y^{n}\right)=$ $\lim _{t \rightarrow \infty} E\left(Y^{n}(t)\right), n \geq 1$ dir. Ek olarak $Y(t)$ sürecinin standartlaştırılmış hali olarak $\tilde{Y}(t)=$ $Y(t)-s$ olarak tanımlanmıştır. Bu durumda

$$
\begin{gathered}
E\left(\tilde{Y}^{n}\right)=\lim _{t \rightarrow \infty} E\left(\tilde{Y}^{n}(t)\right) ; \tilde{\zeta}_{n}=\zeta_{n}-s \\
n=1,2,3, \ldots
\end{gathered}
$$

olacaktır. Khaniyev vd. (2013) çalışmasında $t \rightarrow \infty$ durumunda $\tilde{Y}(t)$ sürecinin ergodik dağılım fonksiyonunun $n$. mertebeden momentleri için kesin formül,

$$
\begin{gathered}
E\left(\tilde{Y}^{n}\right)=\frac{n}{E(U(\tilde{\zeta}))} \int_{0}^{2 a} v^{n-1} E\left(U \left(\widetilde{\zeta_{1}}\right.\right. \\
-v)) d v
\end{gathered}
$$

biçiminde verilmiştir. Burada $\tilde{\zeta}=\zeta-s, a \equiv$ $(S-s) / 2, \quad$ ve $U(x), \quad\left\{\eta_{n}\right\}, n \geq 1$ rasgele değişkenleri tarafından üretilen yenileme fonksiyonudur. 
$Y(t)$ süreci Önerme 3.1.'de verildiği gibi genel ergodik teoreminin şartlarını sağladığı görülmektedir.

Önerme 3.1. (Khaniyev vd., 2013) $Y(t)$ süreci aşağıdaki koşullar altında ergodiktir:

1) $0<E\left(\xi_{1}\right)<\infty$.

2) $E\left(\eta_{1}\right)>0$.

3) $\eta_{1}$ aritmetik olmayan bir rasgele değişkendir.

4) Markov zincirini oluşturan $\left\{\zeta_{n}\right\}$ rasgele değişkenleri $\pi(z)$ dağılım fonksiyonu ile $[s, S]$ aralığında üçgensel dağılıma sahiptir, yani

$$
\begin{gathered}
\pi(z) \equiv \lim _{n \rightarrow \infty} P\left\{\zeta_{n} \leq z\right\}=\int_{S}^{z} p(x) d x \\
p(x)= \begin{cases}\frac{4(x-s)}{(S-s)^{2}}, & s \leq x \leq \frac{S+s}{2} \\
\frac{4(S-x)}{(S-s)^{2}}, & \frac{S+s}{2} \leq x \leq S\end{cases}
\end{gathered}
$$

dır. Bu çalışmada müdahaleyi ifade eden $\zeta_{n}, n \geq$ 1 rasgele değişkenlerinin üçgensel dağılıma sahip olması varsayımı aşağıdaki iki nedene

dayandırılarak yapılmıştır (Khaniyev vd., 2013):

1) Her bir periyodun sonunda sistem $\zeta_{n}, n \geq 1$ müdahalesi ile başlamaktadır. Örneğin ikinci periyod için başlangıç stok seviyesi $\zeta_{1}$ dir. Burada $\zeta_{1}$ başlangıç seviyesinin, stok kontrol seviyesi olan "s" seviyesine çok yakın olması tercih edilmez. Eğer $\zeta_{1}$, "s" seviyesine çok yakın bir değer alır ise, sistem çok kısa bir süre içinde yeniden başlamış demektir ve bu da pratikte tercih edilir bir durum değildir. Sistemin her periyodunun çok kısa bir süre içinde sonlanıp yeniden başlaması siparişlerin sayısını, böylece de sipariş maliyetini artıracaktır. Dolayısıyla, $\zeta_{1}$ müdahale rasgele değişkeni, "s" stok kontrol seviyesine çok düşük bir olasılık ile yakın olacak biçimde belirlenmelidir.

2) Yeniden başlama seviyesi olan $\zeta_{1}$, aynı zamanda $S$ maksimum stok seviyesine de çok yakın olmamalıdır. $\zeta_{1}$ 'in "S" seviyesine çok yakın olması ortalama stok seviyesinin artmasina neden olur ki bu da stoklama maliyet tutarının artması demektir. Başka bir deyişle, $\zeta_{1}$ müdahale rasgele değişkeni aynı zamanda " $\mathrm{S}$ " maksimum stok seviyesine çok düşük bir olasılık ile yakın olacak biçimde belirlenmelidir.

$\zeta_{1}$ rasgele değişkeni için $(s, S)$ aralığında seçilmiş olan üçgensel dağıllım bu iki kriteri de sağladığından $(\mathrm{s}, \mathrm{S})$ tipli yarı-Markov envanter modellerde müdahaleyi ifade etmek için en uygun dağılımdır.

Önerme 3.2. (Khaniyev vd., 2013) Önerme 3.1'in şartları sağlansın. $\mathrm{E}\left(\eta_{1}^{3}\right)<+\infty$ olduğunda $\tilde{Y}(t) \equiv Y(t)-s$, sürecinin ergodik dağ 1 lımının $\mathrm{n}$. momenti $(\mathrm{n}=1,2,3, .) \quad. a \rightarrow \infty$ için aşağıdaki gibi yazılabilir:

$E\left(\tilde{Y}^{n}\right)=\widetilde{M}^{(k) *}=k n_{1} a^{n}+k n_{2} c m_{1} a^{n-1}-m_{1}^{2}\left[A k n_{3}+c^{2} k n_{2}\right] a^{n-2}+o\left(a^{n-2}\right)$

dir. Burada

$k n_{1}=\frac{2^{n+3}-2}{(n+1)(n+2)(n+3)} ; k n_{2}=\frac{(n+1) 2^{n+2}-2(n+2)}{(n+1)(n+2)(n+3)} ;$

$k n_{3}=\frac{2^{n+1}-2}{n+1} ; c=\frac{m_{2}}{2 m_{1}^{2}} ; A=\frac{2 m_{3} m_{1}-3 m_{2}^{2}}{12 m_{1}^{4}} ; m_{k}=E\left(\eta_{1}^{k}\right) ; k=1,2,3 ; a=\frac{S-s}{2}$

dir. Ayrıca, $a=(S-s) / 2 \rightarrow \infty$ olduğundan $S$ nin büyük değerleri için (3.1) ifadesi elde edilmiştir.

\section{4. Üçgensel Müdahaleli Yarı-Markov (s,S) Tipli Modellerin Momentlerinin Tahmini ve İstatistiksel Özellikleri}

Bu bölümde, (3.1)' deki $m_{1}, m_{2}$ ve $m_{3}$ nin tahmin edicilerini kullanarak sürecin ergodik dağılımı için tahmin ediciler aşağıdaki gibi verilebilir:
Burada $\bar{X}=\widehat{m}_{1}=\sum_{i}^{n} \eta_{i} / n, \overline{X^{2}}=\widehat{m}_{2}=\sum_{i}^{n} \eta_{i}^{2} / n$ ve $\overline{X^{3}}=\widehat{m}_{3}=\sum_{i}^{n} \eta_{i}^{3} / n$ dir ve $m_{1}, m_{2}$ ve $m_{3}$ tahmin edicileri $\eta_{1}, \eta_{2}, \ldots, \eta_{3}$ rasgele örneklemine dayanmaktadır. Şimdi sirasiyla (4.1)' deki $\widehat{\widehat{M}}^{(k) *}$ tahmin edicisi için asimptotik yansızlık, tutarlılık ve asimptotik normallik gibi istatistiksel özellikler incelenecektir. 


$$
\begin{aligned}
\widehat{\widetilde{M}}^{(k) *} & =k n_{1} a^{n}+k n_{2} c \bar{X} a^{n-1}-\bar{X}^{2}\left[A k n_{3}+c^{2} k n_{2}\right] a^{n-2} \\
& =k n_{1} a^{n}+k n_{2} \frac{\overline{X^{2}}}{2 \bar{X}^{2}} \bar{X} a^{n-1}-\bar{X}^{2}\left[\frac{2 \overline{X^{3}} \bar{X}-3\left(\overline{X^{2}}\right)^{2}}{12 \bar{X}^{4}} k n_{3}+\frac{\left(\overline{X^{2}}\right)^{2}}{4 \bar{X}^{4}} k n_{2}\right] a^{n-2} \\
& =k n_{1} a^{n}+k n_{2} \frac{\overline{X^{2}}}{2 \bar{X}} a^{n-1}-\left[\left(\frac{\overline{X^{3}}}{6 \bar{X}}-\frac{\left(\overline{X^{2}}\right)^{2}}{4 \bar{X}^{2}}\right) k n_{3}+\frac{\left(\bar{X}^{2}\right)^{2}}{4 \bar{X}^{2}} k n_{2}\right] a^{n-2} .
\end{aligned}
$$

Bunun için öncelikle Önerme 4.1, Önerme 4.2, Önerme 4.3 ve Önerme 4.4' e ihtiyaç duyulacaktır.

Önerme 4.1. (Gökpınar, 2016b) $X_{1}, X_{2}, \ldots, X_{n}$ bağımsız ve aynı $\mathrm{F}$ dağılım fonksiyonuna sahip rasgele değişkenler dizisi ve $m_{i} \quad(\mathrm{i}=1, \ldots)$ dağılımın i-inci başlangıç momenti olmak üzere $\bar{X}$ ve $\overline{X^{2}}$ istatistiklerinin varyansı aşağıdaki gibidir:

$\operatorname{Var}(\bar{X})=\left(\frac{m_{2}-m_{1}^{2}}{n}\right)$

$\operatorname{Var}\left(\overline{X^{2}}\right)=\left(\frac{m_{4}-m_{2}^{2}}{n}\right)$

$\bar{X}$ ve $\overline{X^{2}}$ istatistiklerinin varyansına benzer şekilde $\overline{X^{3}}$ istatistiğinin varyansı Önerme $4.2^{\prime} \mathrm{de}$ hesaplanabilir.

Önerme 4.2. $\overline{X^{3}}$ istatistiğinin varyansı aşağıdaki gibidir:

$\operatorname{Var}\left(\overline{X^{3}}\right)=\left(\frac{m_{6}-m_{3}^{2}}{n}\right)$

İspat: $\overline{X^{3}}$ istatistiğinin varyans1,

$$
\begin{aligned}
\operatorname{Var}\left(\overline{X^{3}}\right)=E\left(\overline{X^{3}}-m_{3}\right)^{2} \\
\quad=E\left[\left(\overline{X^{3}}\right)^{2}\right]-\left[E\left(\overline{X^{3}}\right)\right]^{2}
\end{aligned}
$$

olup eşitliğin sağındaki terimler sırasıyla açııırsa,

$$
\begin{aligned}
E\left[\left(\overline{X^{3}}\right)^{2}\right]=E & {\left[\left(\frac{X_{1}^{3}+X_{2}^{3}+\cdots+X_{n}^{3}}{n}\right)^{2}\right] } \\
& =\frac{1}{n^{2}} E\left(\sum_{i=1}^{n} X_{i}^{6}\right. \\
& \left.+2 \sum_{i<j} \sum^{3} X_{i}^{3} X_{j}^{3}\right)
\end{aligned}
$$

$$
\begin{gathered}
=\frac{1}{n^{2}}\left(n m_{6}+n(n-1) m_{3}^{2}\right) \\
=\frac{m_{6}}{n}+m_{3}^{2}-\frac{m_{3}^{2}}{n}
\end{gathered}
$$

ve

$$
\left[E\left(\overline{X^{3}}\right)\right]^{2}=m_{3}^{2}
$$

elde edilir. Dolayısıyla, (4.4) ispatlanmış olur.

Önerme 4.3. (Gökpınar vd., 2015a) $X_{1}, X_{2}, \ldots, X_{n}$ bağımsız ve aynı $\mathrm{F}$ dağılım fonksiyonuna sahip rasgele değişkenler dizisi ve $m_{i}(\mathrm{i}=1, \ldots)$ dağılımın $\mathrm{i}$-inci başlangıç momenti olsun. $\overline{X^{k}}=\sum_{i=1}^{n} X_{i}^{k} / n \quad(\mathrm{k}=1,2, \ldots)$ ve $\overline{X^{m}}=$ $\sum_{i=1}^{n} X_{i}^{m} / n(\mathrm{~m}=1,2, \ldots)$ sirasiyla $\mathrm{k}$-1nc1 ve m-inci örnek momentleri olmak üzere, $\overline{X^{k}} \mathrm{ve} \overline{X^{m}}$ istatistiklerinin kovaryans1 $\operatorname{Cov}\left(\overline{X^{k}}, \overline{X^{m}}\right)=$ $\left(m_{k+m}-m_{k} m_{m}\right) \backslash n$ şeklindedir. Buna göre, $\mathrm{k}$ ve $\mathrm{m}$ ' nin özel durumlarına göre aşağıdaki sonuçlar elde edilir.

$\operatorname{Cov}\left(\bar{X}, \overline{X^{2}}\right)=\left(\frac{m_{3}-m_{1} m_{2}}{n}\right)$

$\operatorname{Cov}\left(\overline{X^{2}}, \overline{X^{3}}\right)=\left(\frac{m_{5}-m_{2} m_{3}}{n}\right)$

$\operatorname{Cov}\left(\bar{X}, \overline{X^{3}}\right)=\left(\frac{m_{4}-m_{1} m_{3}}{n}\right)$

Önerme 4.4. $\quad \overline{X^{2}} / \bar{X}, \quad \overline{X^{3}} / \bar{X}, \quad\left(\overline{X^{2}}\right)^{2} / \bar{X}^{2}$ istatistiklerinin beklenen değerleri aşağıdaki gibi elde edilir:

$$
\begin{aligned}
& E(\overline{\bar{X}} \overline{\bar{X}}) \cong \frac{m_{2}}{m_{1}}+\left(\frac{m_{2}-m_{1}^{2}}{n}\right)\left(\frac{m_{2}}{m_{1}^{3}}\right)-\left(\frac{m_{3}-m_{1} m_{2}}{n m_{1}^{2}}\right) \\
& E\left(\frac{\overline{X^{3}}}{\bar{X}}\right) \cong \frac{m_{3}}{m_{1}}+\frac{m_{3}}{m_{1}^{3}}\left(\frac{m_{2}-m_{1}^{2}}{n}\right)-\frac{1}{m_{1}^{2}}\left(\frac{m_{4}-m_{3} m_{1}}{n}\right)
\end{aligned}
$$


$E\left(\frac{\left(\overline{X^{2}}\right)^{2}}{\bar{X}^{2}}\right) \cong \frac{m_{2}^{2}}{m_{1}^{2}}+\left(\frac{m_{2}-m_{1}^{2}}{n}\right)\left(\frac{3 m_{2}^{2}}{m_{1}^{4}}\right)+$
$\left(\frac{m_{4}-m_{2}^{2}}{n}\right)\left(\frac{1}{m_{1}^{2}}\right)-\left(\frac{m_{3}-m_{1} m_{2}}{n}\right)\left(\frac{4 m_{2}}{m_{1}^{3}}\right)$
İspat: $g\left(\bar{X}, \overline{X^{2}}\right)=\overline{X^{2}} / \bar{X}$ fonksiyonunu ele alalım. $\mathrm{Bu}$ fonksiyonun $m_{1}$ ve $m_{2}$ noktasindaki Taylor açılımı aşağıdaki gibidir:

$$
\begin{aligned}
& \frac{\overline{X^{2}}}{\bar{X}}=g\left(m_{1}, m_{2}\right)+\left(\bar{X}-m_{1}\right)\left(-\frac{m_{2}}{m_{1}^{2}}\right)+\left(\overline{X^{2}}-m_{2}\right)\left(\frac{1}{m_{1}}\right)+\left(\bar{X}-m_{1}\right)^{2}\left(\frac{m_{2}}{m_{1}^{3}}\right)+\left(\bar{X}-m_{1}\right)\left(\overline{X^{2}}-\right. \\
& \left.m_{2}\right)\left(-\frac{1}{m_{1}^{2}}\right)+R \\
& \quad=\frac{m_{2}}{m_{1}}-\left(\bar{X}-m_{1}\right) \frac{m_{2}}{m_{1}^{2}}+\frac{\left(\overline{X^{2}}-m_{2}\right)}{m_{1}}+\left(\bar{X}-m_{1}\right)^{2} \frac{m_{2}}{m_{1}^{3}}-\left(\bar{X}-m_{1}\right)\left(\overline{X^{2}}-m_{2}\right) \frac{1}{m_{1}^{2}}+R .
\end{aligned}
$$

Burada $\overline{X^{2}} / \bar{X}$ istatistiğinin beklenen değeri (4.2) ve (4.5) kullanılarak

$$
\begin{aligned}
& E\left(\frac{\overline{X^{2}}}{\bar{X}}\right)=\frac{m_{2}}{m_{1}}+E\left(\bar{X}-m_{1}\right)\left(-\frac{m_{2}}{m_{1}^{2}}\right)+E\left(\overline{X^{2}}-m_{2}\right)\left(\frac{1}{m_{1}}\right)+E\left(\bar{X}-m_{1}\right)^{2}\left(\frac{m_{2}}{m_{1}^{3}}\right)+E\left[( \overline { X } - m _ { 1 } ) \left(\overline{X^{2}}-\right.\right. \\
& \left.\left.m_{2}\right)\right]\left(-\frac{1}{m_{1}^{2}}\right)+E(R) \\
& \cong \frac{m_{2}}{m_{1}}+\operatorname{Var}(\bar{X})\left(\frac{m_{2}}{m_{1}^{3}}\right)+\operatorname{Cov}\left(\bar{X}, \overline{X^{2}}\right)\left(-\frac{1}{m_{1}^{2}}\right) \cong \frac{m_{2}}{m_{1}}+\left(\frac{m_{2}-m_{1}^{2}}{n}\right)\left(\frac{m_{2}}{m_{1}^{3}}\right)-\left(\frac{m_{3}-m_{1} m_{2}}{n m_{1}^{2}}\right)
\end{aligned}
$$

olarak elde edilir. Burada Büyük Sayılar Kanuna göre $\bar{X} \stackrel{p}{\rightarrow} m_{1}$ ve $\overline{X^{2}} \stackrel{p}{\rightarrow} m_{2}$ olduğundan kalan terimin beklenen değeri $(E(R)), n \rightarrow \infty$ iken 0 ' a gider. Diğer beklenen değerler (4.9) ve (4.10) da aynı yöntemle, Önerme 4.1, Önerme 4.2 ve Önerme 4.3 yardımıyla bulunabilir.

$\widehat{\widetilde{M}}^{(k) *}$ tahmin edicisinin asimptotik yansızlık özelliği Teorem 4.1' de verilecektir.

Teorem 4.1. $m_{4}=E\left(\eta_{1}^{4}\right)<\infty$ olmak üzere,

$\lim _{n \rightarrow \infty} E\left(\widehat{\widetilde{M}}^{(k) *}\right)=k n_{1} a^{n}+\frac{k n_{2} m_{2}}{2 m_{1}} a^{n-1}-$ $m_{1}^{2}\left[\left(\frac{2 m_{3} m_{1}-3 m_{2}^{2}}{12 m_{1}^{4}}\right) k n_{3}+\left(\frac{m_{2}}{2 m_{1}^{2}}\right)^{2} k n_{2}\right] a^{n-2}$

dir. Yani, $\widehat{\widetilde{M}}^{(k) *}, \widetilde{M}^{(k) *}$ nin asimptotik yansız tahmin edicisidir.

İspat: $\widehat{\widehat{M}}^{(k) *}$ nin beklenen değeri,

$$
\begin{aligned}
& E\left(\widehat{\widetilde{M}}^{(k) *}\right)=k n_{1} a^{n}+k n_{2} E\left(\frac{\overline{X^{2}}}{2 \bar{X}}\right) a^{n-1} \\
& -\left[\left(E\left(\frac{\overline{X^{3}}}{6 \bar{X}}\right)-E\left(\frac{\left(\overline{X^{2}}\right)^{2}}{4 \bar{X}^{2}}\right)\right) k n_{3}+\right. \\
& \left.E\left(\frac{\left({\overline{X^{2}}}^{2}\right.}{\overline{4 X^{2}}}\right) k n_{2}\right] a^{n-2}
\end{aligned}
$$

olur. (4.8)-(4.10)' daki eşitlikler $E\left(\widehat{\widetilde{M}}^{(k) *}\right)$ ' de yerine yazılırsa,

$E\left(\widehat{\widetilde{M}}^{(k) *}\right)=$

$k n_{1} a^{n}+\frac{k n_{2}}{2}\left[\frac{m_{2}}{m_{1}}+\left(\frac{m_{2}-m_{1}^{2}}{n}\right)\left(\frac{m_{2}}{m_{1}^{3}}\right)-\right.$

$\left.\left(\frac{m_{3}-m_{1} m_{2}}{n m_{1}^{2}}\right)\right] a^{n-1}$

$$
\begin{gathered}
-\frac{1}{6}\left\{\frac{m_{3}}{m_{1}}+\frac{m_{3}}{m_{1}^{3}}\left(\frac{m_{2}-m_{1}^{2}}{n}\right)-\right. \\
\left.\frac{1}{m_{1}^{2}}\left(\frac{m_{4}-m_{1} m_{3}}{n}\right)\right\} k n_{3} a^{n-2} \\
+\frac{1}{4}\left\{\frac{m_{2}^{2}}{m_{1}^{2}}+3\left(\frac{m_{2}-m_{1}^{2}}{n}\right)\left(\frac{m_{2}^{2}}{m_{1}^{4}}\right)+\right. \\
\left.\left(\frac{m_{4}-m_{2}^{2}}{n}\right)\left(\frac{1}{m_{1}^{2}}\right)-4\left(\frac{m_{3}-m_{1} m_{2}}{n}\right)\left(\frac{m_{2}}{m_{1}^{3}}\right)\right\} k n_{3} a^{n-2} \\
-\frac{1}{4}\left\{\frac{m_{2}^{2}}{m_{1}^{2}}+3\left(\frac{m_{2}-m_{1}^{2}}{n}\right)\left(\frac{m_{2}^{2}}{m_{1}^{4}}\right)+\right. \\
\left.\left(\frac{m_{4}-m_{2}^{2}}{n}\right)\left(\frac{1}{m_{1}^{2}}\right)-4\left(\frac{m_{3}-m_{1} m_{2}}{n}\right)\left(\frac{m_{2}}{m_{1}^{3}}\right)\right\} k n_{2} a^{n-2}
\end{gathered}
$$

elde edilir. Ve böylece,

$$
\begin{aligned}
& \lim _{n \rightarrow \infty} E\left(\widehat{\widetilde{M}}^{(k) *}\right)=k n_{1} a^{n}+k n_{2} \frac{m_{2}}{2 m_{1}} a^{n-1}- \\
& m_{1}^{2}\left[\left(\frac{2 m_{3} m_{1}-3 m_{2}^{2}}{12 m_{1}^{4}}\right) k n_{3}+\frac{m_{2}^{2}}{4 m_{1}^{4}} k n_{2}\right] a^{n-2} \\
& \text { hesaplanir. }
\end{aligned}
$$


Şimdi $\widehat{\widetilde{M}}^{(k) *}$ tahmin edicisinin tutarlı bir tahmin edici olduğunu göstermeden önce varyans1 bulunacaktır. Bunun ile ilgili yardımc1 teorem aşağıdadır.

Yardımcı Teorem 4.1. $m_{6}=E\left(\eta_{1}^{6}\right)<\infty$ olmak üzere $\widehat{\widehat{M}}^{(k) *}$, nın varyansı,

$$
\operatorname{Var}\left(\widehat{\widetilde{M}}^{(k) *}\right)=\frac{X}{n}+\frac{Y}{n^{2}}
$$

dir. Burada $X=A+B+C+D+E+F \quad$ ve $Y=G+H+I+J+K+L$ olup sirasiyla,

$$
\begin{aligned}
& A=\left(k n_{2} a^{n-1}\right)^{2}\left(\frac{m_{2}^{3}}{4 m_{1}^{4}}+\frac{m_{4}}{4 m_{1}^{2}}-\frac{m_{2} m_{3}}{2 m_{1}^{3}}\right) \\
& B=\left(k n_{2} a^{n-2}\right)^{2}\left(\frac{m_{2}^{5}}{4 m_{1}^{6}}+\frac{3 m_{4} m_{2}^{2}}{8 m_{1}^{4}}-\frac{m_{2}^{3} m_{3}}{2 m_{1}^{5}}\right) \\
& C=\left(k n_{3} a^{n-2}\right)^{2}\left\{\frac{119 m_{2} m_{3}^{2}}{18 m_{1}^{4}}+\frac{m_{6}}{36 m_{1}^{2}}-\frac{5 m_{3} m_{4}}{36 m_{1}^{3}}+\right. \\
& \frac{m_{2}^{5}}{4 m_{1}^{6}}+\frac{3 m_{2}^{4}}{8 m_{1}^{4}}+\frac{13 m_{4} m_{2}^{2}}{24 m_{1}^{4}}-\frac{3 m_{2}^{4}}{4 m_{1}^{6}}-\frac{23 m_{2}^{3} m_{3}}{m_{1}^{5}}-\frac{m_{2}^{2} m_{3}}{6 m_{1}^{3}}- \\
& \left.\frac{m_{2} m_{5}}{6 m_{1}^{3}}\right\}
\end{aligned}
$$$$
D=k n_{2} k n_{3}\left(a^{n-2}\right)^{2}\left(-\frac{m_{2}^{5}}{2 m_{1}^{6}}-\frac{11 m_{2}^{2} m_{4}}{12 m_{1}^{4}}+\right.
$$$$
\left.\frac{7 m_{2}^{3} m_{3}}{6 m_{1}^{5}}+\frac{m_{2}^{2} m_{3}}{6 m_{1}^{3}}+\frac{m_{3} m_{4}}{12 m_{1}^{3}}-\frac{m_{2} m_{3}^{2}}{6 m_{1}^{4}}+\frac{m_{2} m_{5}}{6 m_{1}^{3}}\right)
$$$$
E=k n_{2} k n_{3} a^{n-1} a^{n-2}\left\{\frac{3 m_{2}^{4}}{4 m_{1}^{2}}+\frac{17 m_{2} m_{4}}{6 m_{1}^{3}}-\right.
$$$$
\frac{29 m_{2}^{2} m_{3}}{12 m_{1}^{4}}+\frac{m_{3}^{2}}{6 m_{1}^{3}}-\frac{m_{5}}{6 m_{1}^{2}}+\frac{m_{3}^{2}}{6 m_{1}^{2}}-\frac{5 m_{2} m_{3}}{12 m_{1}^{2}}-\frac{m_{2}^{4}}{4 m_{1}^{4}}+
$$$$
\left.\frac{m_{2}^{2}}{4 m_{1}^{2}}+\frac{m_{2} m_{3}}{4 m_{1}^{3}}\right\}
$$$$
F=\left(k n_{2}\right)^{2} a^{n-1} a^{n-2}\left(-\frac{m_{2}^{2}}{4 m_{1}^{2}}-\frac{m_{2} m_{3}}{4 m_{1}^{3}}+\frac{m_{2} m_{3}}{4 m_{1}^{2}}-\right.
$$$$
\left.\frac{3 m_{2}^{4}}{4 m_{1}^{5}}-\frac{m_{2} m_{4}}{2 m_{1}^{3}}+\frac{5 m_{2}^{2} m_{3}}{4 m_{1}^{4}}\right)
$$

$$
\begin{aligned}
& G=\left(k n_{2} a^{n-1}\right)^{2}\left\{\frac{m_{2}^{2} m_{3}}{2 m_{1}^{5}}-\frac{m_{3}^{2}}{4 m_{1}^{4}}-\frac{m_{2}^{4}}{4 m_{1}^{6}}\right\} \\
& H=\left(k n_{2} a^{n-2}\right)^{2}\left(-\frac{9 m_{2}^{6}}{16 m_{1}^{8}}-\frac{m_{4}^{2}}{16 m_{1}^{4}}-\frac{m_{2}^{2} m_{3}^{2}}{m_{1}^{6}}-\right. \\
& \left.\frac{3 m_{2}^{3} m_{4}}{8 m_{1}^{6}}-\frac{m_{2}^{2} m_{4}}{8 m_{1}^{4}}+\frac{3 m_{2}^{4} m_{3}}{2 m_{1}^{7}}+\frac{m_{2} m_{3} m_{4}}{2 m_{1}^{5}}\right)
\end{aligned}
$$$$
I=\left(k n_{3} a^{n-2}\right)^{2}\left(\frac{m_{2}^{2} m_{3}}{4 m_{1}^{3}}-\frac{49 m_{2}^{2} m_{3}^{2}}{36 m_{1}^{6}}-\frac{25 m_{4}^{2}}{144 m_{1}^{4}}-\right.
$$$$
\left.\frac{m_{2} m_{3} m_{4}}{36 m_{1}^{5}}-\frac{9 m_{2}^{6}}{16 m_{1}^{8}}-\frac{5 m_{2}^{3} m_{4}}{8 m_{1}^{6}}-\frac{m_{2}^{2} m_{4}}{8 m_{1}^{4}}-\frac{5 m_{2}^{4} m_{3}}{4 m_{1}^{7}}\right)
$$

$J=k n_{2} k n_{3}\left(a^{n-2}\right)^{2}\left\{\frac{9 m_{2}^{6}}{8 m_{1}^{8}}+\frac{5 m_{4}^{2}}{24 m_{1}^{4}}+\frac{7 m_{2}^{2} m_{3}^{2}}{3 m_{1}^{6}}+\right.$

$\frac{m_{2}^{3} m_{4}}{m_{1}^{6}}-\frac{13 m_{2}^{4} m_{3}}{4 m_{1}^{7}}-\frac{17 m_{2} m_{3} m_{4}}{12 m_{1}^{5}}+\frac{m_{2}^{2} m_{4}}{4 m_{1}^{4}}+\frac{m_{2}^{3} m_{3}}{4 m_{1}^{5}}-$

$\left.\frac{m_{2}^{2} m_{3}}{4 m_{1}^{3}}\right\}$

$K=k n_{2} k n_{3} a^{n-1} a^{n-2}\left\{\frac{23 m_{2}^{3} m_{3}}{12 m_{1}^{6}}-\frac{m_{2}^{2} m_{3}}{6 m_{1}^{4}}-\right.$

$\frac{7 m_{2} m_{3}^{2}}{6 m_{1}^{5}}+\frac{7 m_{2} m_{3}^{2}}{6 m_{1}^{4}}-\frac{5 m_{4} m_{2}^{2}}{12 m_{1}^{5}}+\frac{5 m_{2} m_{4}}{12 m_{1}^{3}}+\frac{5 m_{3} m_{4}}{12 m_{1}^{4}}-$

$\left.\frac{5 m_{3} m_{4}}{12 m_{1}^{3}}-\frac{3 m_{2}^{5}}{4 m_{1}^{7}}+\frac{3 m_{2}^{4}}{4 m_{1}^{5}}-\frac{3 m_{3} m_{2}^{3}}{4 m_{1}^{5}}\right\}$

$L=\left(k n_{2}\right)^{2} a^{n-1} a^{n-2}\left\{\frac{3 m_{2}^{5}}{4 m_{1}^{7}}-\frac{3 m_{2}^{4}}{4 m_{1}^{5}}-\frac{7 m_{2}^{3} m_{3}}{4 m_{1}^{6}}+\right.$

$\frac{3 m_{2}^{3} m_{3}}{4 m_{1}^{5}}+\frac{m_{2}^{2} m_{4}}{4 m_{1}^{5}}-\frac{m_{2} m_{4}}{4 m_{1}^{3}}-\frac{m_{3} m_{4}}{4 m_{1}^{4}}-\frac{m_{3} m_{4}}{4 m_{1}^{3}}+\frac{m_{2} m_{3}^{2}}{m_{1}^{5}}-$

$\left.\frac{m_{3}^{2} m_{2}}{m_{1}^{4}}\right\}$

dir.

İspat: $\widehat{\widetilde{M}}^{(k) *}$ 'nın varyansını hesaplamak için sirasiyla $E\left[\left(\widehat{\widetilde{M}}^{(k) *}\right)^{2}\right] \quad$ ve $\left[E\left(\widehat{\widetilde{M}}^{(k) *}\right)\right]^{2}$ elde edilecektir. Bunun için Önerme 4.4'deki denklemler kullanılacaktır. 


$$
\begin{aligned}
& E\left[\left(\widehat{\widetilde{M}}^{(k) *}\right)^{2}\right]=E\left[\left(k n_{1} a^{n}\right)^{2}+\left(\frac{k n_{2} a^{n-1}}{2}\right)^{2}\left(\frac{\overline{X^{2}}}{\bar{X}}\right)^{2}+k n_{1} k n_{2} a^{n-1} a^{n}\left(\frac{\overline{X^{2}}}{\bar{X}}\right)\right. \\
& +\frac{\left(k n_{3} a^{n-2}\right)^{2}}{36}\left(\frac{\overline{X^{3}}}{\bar{X}}\right)^{2}+\frac{\left(k n_{3} a^{n-2}\right)^{2}}{16}\left(\frac{\overline{X^{2}}}{\bar{X}}\right)^{4}-\frac{\left(k n_{3} a^{n-2}\right)^{2}}{12}\left(\frac{\overline{X^{3}}}{\bar{X}}\right)\left(\frac{\overline{X^{2}}}{\bar{X}}\right)^{2} \\
& +\frac{\left(k n_{2} a^{n-2}\right)^{2}}{16}\left(\frac{\overline{X^{2}}}{\bar{X}}\right)^{4}+\frac{k n_{2} k n_{3}\left(a^{n-2}\right)^{2}}{12}\left(\frac{\overline{X^{3}}}{\bar{X}}\right)\left(\frac{\overline{X^{2}}}{\bar{X}}\right)^{2}-\frac{k n_{2} k n_{3}\left(a^{n-2}\right)^{2}}{8}\left(\frac{\overline{X^{2}}}{\bar{X}}\right)^{4} \\
& -\frac{k n_{1} k n_{3} a^{n-2} a^{n}}{3}\left(\frac{\overline{X^{3}}}{\bar{X}}\right)+\frac{k n_{1} k n_{3} a^{n-2} a^{n}}{2}\left(\frac{\overline{X^{2}}}{\bar{X}}\right)^{2}-\frac{k n_{1} k n_{2} a^{n-2} a^{n}}{2}\left(\frac{\overline{X^{2}}}{\bar{X}}\right)^{2} \\
& -\frac{k n_{2} k n_{3} a^{n-2} a^{n-1}}{6}\left(\frac{\overline{X^{3}}}{\bar{X}}\right)\left(\frac{\overline{X^{2}}}{\bar{X}}\right)+\frac{k n_{2} k n_{3} a^{n-2} a^{n-1}}{4}\left(\frac{\overline{X^{2}}}{\bar{X}}\right)^{3} \\
& \left.-\frac{\left(k n_{2}\right)^{2} a^{n-2} a^{n-1}}{4}\left(\frac{\overline{X^{2}}}{\bar{X}}\right)^{3}\right]
\end{aligned}
$$

ve

$$
\begin{aligned}
{\left[E\left(\widehat{\widetilde{M}}^{(k) *}\right)\right]^{2}=} & \left\{k n_{1} a^{n}+\frac{k n_{2}}{2}\left[\frac{m_{2}}{m_{1}}+\left(\frac{m_{2}-m_{1}^{2}}{n}\right)\left(\frac{m_{2}}{m_{1}^{3}}\right)-\left(\frac{m_{3}-m_{1} m_{2}}{n m_{1}^{2}}\right)\right] a^{n-1}\right. \\
& -\left[\left(\left\{\frac{m_{3}}{6 m_{1}}+\frac{m_{3}}{m_{1}^{3}}\left(\frac{m_{2}-m_{1}^{2}}{6 n}\right)-\left(\frac{m_{4}-m_{1} m_{3}}{6 n m_{1}^{2}}\right)\right\}\right.\right. \\
& \left.+\left\{\frac{m_{2}^{2}}{4 m_{1}^{2}}+3\left(\frac{m_{2}^{3}-m_{1}^{2} m_{2}^{2}}{4 n m_{1}^{4}}\right)+\frac{m_{4}-m_{2}^{2}}{4 m_{1}^{2} n}-\left(\frac{m_{3}-m_{1} m_{2}}{n}\right)\left(\frac{m_{2}}{m_{1}^{3}}\right)\right\}\right) k n_{3} \\
& \left.\left.-\left\{\frac{m_{2}^{2}}{4 m_{1}^{2}}+\frac{3\left(m_{2}^{3}-m_{1}^{2} m_{2}^{2}\right)}{4 n m_{1}^{4}}+\frac{m_{4}-m_{2}^{2}}{4 m_{1}^{2} n}-\frac{\left(m_{3} m_{2}-m_{1} m_{2}^{2}\right)}{n m_{1}^{3}}\right\} k n_{2}\right] a^{n-2}\right\}^{2}
\end{aligned}
$$

dır. Böylece,

$\operatorname{Var}\left(\widehat{\widetilde{M}}^{(k) *}\right)=E\left[\left(\widehat{\widetilde{M}}^{(k) *}\right)^{2}\right]-\left[E\left(\widehat{\widetilde{M}}^{(k) *}\right)\right]^{2}=\frac{X}{n}+\frac{Y}{n^{2}} \quad$ elde edilir.

Teorem 4.2. $m_{6}=E\left(\eta_{1}^{6}\right)<\infty$ olmak üzere $\widehat{\widetilde{M}}^{(k) *}$ tahmin edicisi, $\widetilde{M}^{(k) *}$ için tutarlı bir tahmin edicidir.

İspat: $\widehat{\widetilde{M}}^{(k) *}$ tahmin edicisinin $\widetilde{M}^{(k) *}$ için tutarl 1 bir tahmin edici olduğunu göstermek için

$\lim _{n \rightarrow \infty} P\left(\left|\widetilde{M}^{(k) *}-\widehat{\widetilde{M}}^{(k) *}\right| \geq \varepsilon\right)=0$

olduğunu göstermek gerekir. Markov eşitsizliğinden

$P\left(\left|\widetilde{M}^{(k) *}-\widehat{\widetilde{M}}^{(k) *}\right| \geq \varepsilon\right) \leq \frac{\operatorname{Var}\left(\widehat{\widehat{M}}^{(k) *}\right)}{\varepsilon^{2}}$

ve Yardımcı Teorem 4.1' den $\lim _{n \rightarrow \infty} \operatorname{Var}\left(\widehat{\widetilde{M}}^{(k) *}\right)=0$

olduğundan istenilen sağlanır. Dolayısıyla $\widehat{\widetilde{M}}^{(k) *}$ tahmin edicisi, $\widetilde{M}^{(k) *}$ için tutarlı bir tahmin edicidir denir.

Teorem 4.3. $a \equiv(S-s) / 2 \rightarrow \infty$ ve $n \rightarrow \infty$ iken $\widehat{\widetilde{M}}^{(k) *}-\widetilde{M}^{(k) *} \stackrel{p}{\rightarrow} 0$ dir.

\section{İspat:}

$$
\begin{gathered}
P\left(\left|\widehat{\widetilde{M}}^{(k) *}-\widetilde{M}^{(k)}\right|<\varepsilon\right)=P\left(\mid \widehat{\widetilde{M}}^{(k) *}-\right. \\
\left.\widetilde{M}^{(k) *}+\widetilde{M}^{(k) *}-\widetilde{M}^{(k)} \mid<\varepsilon\right) \\
\geq P\left(\left|\widehat{\widetilde{M}}^{(k) *}-\widetilde{M}^{(k) *}\right|<\frac{\varepsilon}{2} ;\left|\widetilde{M}^{(k) *}-\widetilde{M}^{(k)}\right|\right. \\
\left.<\frac{\varepsilon}{2}\right)
\end{gathered}
$$


olur. Buradan,

$P\left(\left|\widehat{\widetilde{M}}^{(k) *}-\widetilde{M}^{(k) *}\right|<\frac{\varepsilon}{2}\right) \rightarrow 1$

ve

$P\left(\left|\widetilde{M}^{(k) *}-\widetilde{M}^{(k)}\right|<\frac{\varepsilon}{2}\right) \rightarrow 1$

için

$P\left(\left|\widehat{\widetilde{M}}^{(k) *}-\widetilde{M}^{(k)}\right|<\varepsilon\right)=1$ dir. Dolayısiyla $a \equiv(S-s) / 2 \rightarrow \infty$ ve $n \rightarrow \infty$ iken $\widehat{\widehat{M}}^{(k) *}-\widetilde{M}^{(k) *} \stackrel{p}{\rightarrow} 0$ dir.

Tahmin edicinin asimptotik normal dağılıma sahip olduğu Teorem 4.4' de gösterildiği gibidir.

Teorem 4.4. $m_{6}=E\left(\eta_{1}^{6}\right)<\infty$ olmak üzere, $\widehat{\widehat{M}}^{(k) *}$ asimptotik normaldir, yani

$\sqrt{n}\left(\widehat{\widetilde{M}}^{(k) *}-\widetilde{M}^{(k) *}\right) \underset{n \rightarrow \infty}{\longrightarrow} N\left(0, \sigma^{2}\right)$

dir. Burada

$$
\begin{aligned}
\sigma^{2} & =\left(\frac{m_{2}-m_{1}^{2}}{n}\right)\left[-\frac{k n_{2} a^{n-1}}{2} \frac{m_{2}}{m_{1}^{2}}+\frac{k n_{3} a^{n-2}}{6} \frac{m_{3}}{m_{1}^{2}}-\frac{k n_{3} a^{n-2}}{2} \frac{m_{2}^{2}}{m_{1}^{3}}+\frac{k n_{2} a^{n-2}}{2} \frac{m_{2}^{2}}{m_{1}^{3}}\right]^{2} \\
& +\left(\frac{m_{4}-m_{2}^{2}}{n}\right)\left[\frac{k n_{2} a^{n-1}}{2 m_{1}}+\frac{k n_{3} a^{n-2} m_{2}}{2 m_{1}^{2}}-\frac{k n_{2} a^{n-2} m_{2}}{2 m_{1}^{2}}\right]^{2}+\left(\frac{m_{6}-m_{3}^{2}}{n}\right)\left[\frac{k n_{3} a^{n-2}}{-6 m_{1}}\right]^{2} \\
& +2\left(\frac{m_{3}-m_{1} m_{2}}{n}\right)\left(\frac{k n_{3} a^{n-2}}{6} \frac{m_{3}}{m_{1}^{2}}-\frac{k n_{2} a^{n-1}}{2} \frac{m_{2}}{m_{1}^{2}}-\frac{k n_{3} a^{n-2}}{2} \frac{m_{2}^{2}}{m_{1}^{3}}+\frac{k n_{2} a^{n-2}}{2} \frac{m_{2}^{2}}{m_{1}^{3}}\right) \\
& \times\left(\frac{k n_{2} a^{n-1}}{2} \frac{1}{m_{1}}+\frac{k n_{3} a^{n-2}}{2} \frac{m_{2}}{m_{1}^{2}}-\frac{k n_{2} a^{n-2}}{2} \frac{m_{2}}{m_{1}^{2}}\right)+2\left(\frac{m_{4}-m_{1} m_{3}}{n}\right)\left(-\frac{k n_{3} a^{n-2}}{6 m_{1}}\right) \\
& \times\left(\frac{k n_{3} a^{n-2}}{6} \frac{m_{3}}{m_{1}^{2}}-\frac{k n_{2} a^{n-1}}{2} \frac{m_{2}}{m_{1}^{2}}-\frac{k n_{3} a^{n-2}}{2} \frac{m_{2}^{2}}{m_{1}^{3}}+\frac{k n_{2} a^{n-2}}{2} \frac{m_{2}^{2}}{m_{1}^{3}}\right) \\
& \quad+2\left(\frac{m_{5}-m_{2} m_{3}}{n}\right)\left[\left(\frac{k n_{2} a^{n-1}}{2} \frac{1}{m_{1}}+\frac{k n_{3} a^{n-2}}{2} \frac{m_{2}}{m_{1}^{2}}-\frac{k n_{2} a^{n-2}}{2} \frac{m_{2}}{m_{1}^{2}}\right)\left(-\frac{k n_{3} a^{n-2}}{6 m_{1}}\right)\right]
\end{aligned}
$$

dir.

İspat: $\mathrm{Bu}$ teoremin ispatı için Çoklu Delta Yönteminden faydalanıldığında (4.1)-(4.7) denklemlerinden aşağıdaki sonuçlar elde edilir (Casella. ve Berger, 2002).

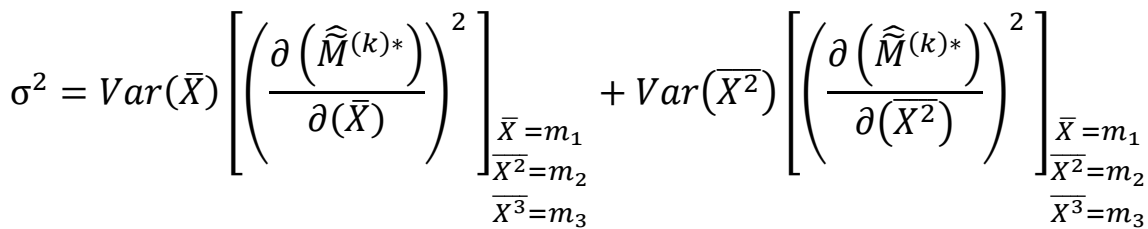

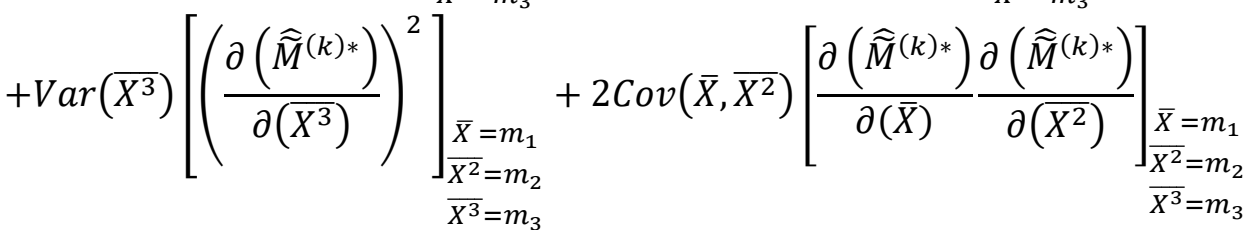

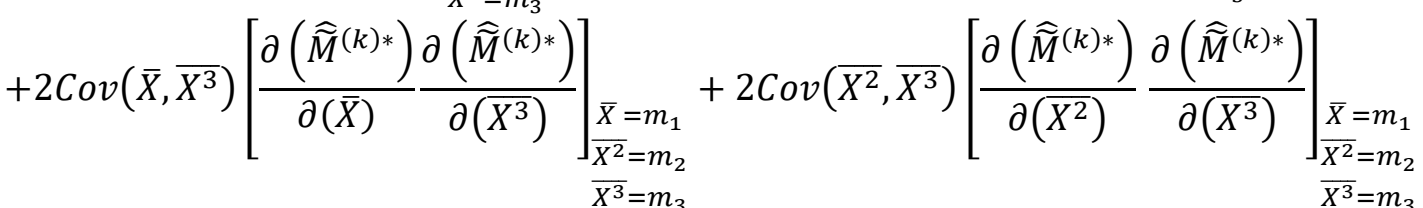

$$
\begin{aligned}
& =\left(\frac{m_{2}-m_{1}^{2}}{n}\right)\left[-\frac{k n_{2} a^{n-1}}{2} \frac{m_{2}}{m_{1}^{2}}+\frac{k n_{3} a^{n-2}}{6} \frac{m_{3}}{m_{1}^{2}}-\frac{k n_{3} a^{n-2}}{2} \frac{m_{2}^{2}}{m_{1}^{3}}+\frac{k n_{2} a^{n-2}}{2} \frac{m_{2}^{2}}{m_{1}^{3}}\right]^{2} \\
& +\left(\frac{m_{4}-m_{2}^{2}}{n}\right)\left[\frac{k n_{2} a^{n-1}}{2} \frac{1}{m_{1}}+\frac{k n_{3} a^{n-2}}{2} \frac{m_{2}}{m_{1}^{2}}-\frac{k n_{2} a^{n-2} m_{2}}{2 m_{1}^{2}}\right]^{2}+\left(\frac{m_{6}-m_{3}^{2}}{n}\right)\left[\frac{k n_{3} a^{n-2}}{-6 m_{1}}\right]^{2}
\end{aligned}
$$




$$
\begin{aligned}
& +2\left(\frac{m_{3}-m_{1} m_{2}}{n}\right)\left(-\frac{k n_{2} a^{n-1}}{2} \frac{m_{2}}{m_{1}^{2}}+\frac{k n_{3} a^{n-2}}{6} \frac{m_{3}}{m_{1}^{2}}-\frac{k n_{3} a^{n-2}}{2} \frac{m_{2}^{2}}{m_{1}^{3}}+\frac{k n_{2} a^{n-2}}{2} \frac{m_{2}^{2}}{m_{1}^{3}}\right) \\
& \times\left(\frac{k n_{2} a^{n-1}}{2} \frac{1}{m_{1}}+\frac{k n_{3} a^{n-2}}{2} \frac{m_{2}}{m_{1}^{2}}-\frac{k n_{2} a^{n-2}}{2} \frac{m_{2}}{m_{1}^{2}}\right)+2\left(\frac{m_{4}-m_{1} m_{3}}{n}\right)\left(-\frac{k n_{3} a^{n-2}}{6} \frac{1}{m_{1}}\right) \\
& \times\left(\frac{k n_{3} a^{n-2}}{6} \frac{m_{3}}{m_{1}^{2}}-\frac{k n_{2} a^{n-1}}{2} \frac{m_{2}}{m_{1}^{2}}-\frac{k n_{3} a^{n-2}}{2} \frac{m_{2}^{2}}{m_{1}^{3}}+\frac{k n_{2} a^{n-2}}{2} \frac{m_{2}^{2}}{m_{1}^{3}}\right) \\
& +2\left(\frac{m_{5}-m_{2} m_{3}}{n}\right)\left[\left(\frac{k n_{2} a^{n-1}}{2} \frac{1}{m_{1}}+\frac{k n_{3} a^{n-2}}{2} \frac{m_{2}}{m_{1}^{2}}-\frac{k n_{2} a^{n-2}}{2} \frac{m_{2}}{m_{1}^{2}}\right)\left(-\frac{k n_{3} a^{n-2}}{6} \frac{1}{m_{1}}\right)\right]
\end{aligned}
$$

olarak hesaplanır.

Böylece,

$\sqrt{n}\left(\widehat{\widetilde{M}}^{(k) *}-\widetilde{M}^{(k) *}\right) \underset{n \rightarrow \infty}{\longrightarrow} N\left(0, \sigma^{2}\right)$

olup, $\widehat{\widetilde{M}}^{(k) *}$ tahmin edicisi asimptotik normal dağılıma sahiptir.

Sonuç olarak üçgensel müdahaleli $(\mathrm{s}, \mathrm{S})$ tipli yarıMarkov envarter modelinin ergodik dağ 1 lımının momentleri için önerilmiş olan tahmin edicisi sirasiyla tutarlilık, asimptotik yansızlı ve asimptotik normallik gibi istatistiksel özelliklere sahip olduğu görülmektedir.

\section{Teşekkür}

Makalenin yazarları olarak, Prof. Dr. Tahir KHANIYEV'e (TOBB Ekonomi ve Teknoloji Üniversitesi, Endüstri Mühendisliği, Ankara, Türkiye) değerli destek ve tavsiyelerinden dolayı teşekkür ederiz.

Ayrıca makale yazarlarından Melek ERIŞ BÜYÜKKAYA, 2211-A Genel Yurt İçi Doktora Burs Programı kapsamındaki burs desteğinden dolayı TÜBİTAK'a teşekkür eder.

\section{Kaynaklar}

Bebbington, M., Davydov, Y. ve Zitikis, R., 2007. Estimating the Renewal Function When the Second Moment is Infinite, Stochastic Models, 23(1), 27-48.

Casella, G. ve Berger, R.L. 2002. Statistical Inference (Second edition). Pacific Grove, CA, Duxbury/ Thomson Learning.

Feller, W., 1971. An Introduction to Probability Theory and Its Application 2, Wiley, New York, $669 \mathrm{p}$.
Frees, E.W., 1986 a. Warranty Analysis and Renewal Function Estimation. Nav. Res. Logist. Q., 33, 361-372.

Frees, E.W., 1986 b. Nonparametric Renewal Function Estimation. Annals of Statistics, 14(4), 1366-1378.

Gökpınar, E., Khaniyev, T. ve Gamgam, H., 2015a. Asymptotic Properties of the Straight Line Estimator for a Renewal Function. Sains Malaysiana. 44(7), 10411051.

Gökpınar, E., Khaniyev, T., Gamgam, H. ve Gökpınar, F., 2015b. A New Estimator for Stationary Distribution of the Inventory Model of Type $(\mathrm{s}, \mathrm{S})$. Gazi University Journal of Science. 28(1), 87-101.

Gökpınar, E., Khaniyev, T., Gamgam, H. ve Gökpınar, F., 2016a. Estimators of the Moments for the Invertory Models of Type $(\mathrm{s}, \mathrm{S})$ with a Discrete Interference of Chance. Journal of the Turkish Statistical Association. Vol. 9, No. 1, pp. 17-28.

Gökpınar, E., Khaniyev, T., Gamgam, H. ve Gökpınar, F., 2018. Estimators of the Moments for the Invertory Models of Type $(\mathrm{s}, \mathrm{S})$. Iranian Journal of Science and Technology, Transactions A: Science. Vol. 42, No. 1, pp. 5-12.

Gökpınar, E., 2016b. Yenileme Sürecinde Tahmin Ediciler ve Uygulamalar1. Doktora Tezi, Gazi Üniversitesi Fen Bilimleri Enstitüsü. Ankara, $111 \mathrm{~s}$.

Grübel, R. ve Pitts, S., 1993. Nonparametric Estimation in Renewal Theory 1: the Empirical Renewal Function. Annals of Statistics, 21(3), 1431-145. 
Guedon, Y. ve Cocozza-Thivent, C., 2003. Nonparametric Estimation of Renewal Processes from Count Data. The Canadian Journal of Statistics, 12(3), 191-223.

Kesemen, T., Bektaş Kamışlık, A., Küçük, Z. ve Şenol, E., 2016. Inventory Model of Type $(\mathrm{s}, \mathrm{S}) \quad$ with Subexponential Weibull Distributed Demand. Journal of the Turkish Statistical Association, 9 (3), 81-92.

Khaniyev, T.A. ve Atalay, K.D., 2010. On the Weak Convergence of the Ergodic Distribution for an Inventory Model of Type $(\mathrm{s}, \mathrm{S})$ ". Hacettepe Journal of Mathematics and Statistics, 9 (4), 599-611.

Khaniyev, T.A., Kokangul, A. ve Aliyev, R.T., 2013. An Asymptotic Approach for a SemiMarkovian Inventory Model of Type $(s, S)$. Applied Stochastic Models in Business and Industry, 29 (5), $439-453$.

Markovich N.M. ve Krieger, U.R., 2006. Nonparametric Estimation of the Renewal Function by Empirical Data. Stochastic Models, 22 (2), 175-199.
Necir, A., Rassoul, A. ve Meraghni, D., 2010. POT-Based Estimation of the Renewal Function of Interoccurrence Times of Heavy-Tailed Risks. Journal of Probability and Statistics, doi:10.1155/2010/965672.

Schneider, H., Lin, B.S. ve O’Cinneide, C., 1990. Comparison of Nonparametric Estimators for the Renewal Function. Applied Statistics, 39(1), 55-61.

Sgibnev, M.S., 1981. On the Renewal Theorem in the Case of Infinite Variance, Sibirskit Mathematicheskit Zhurnal, 22(5), 178-189.

Vardi, Y., 1982. Nonparametric Estimation in Renewal Processes. Annals of Statistics, 10(3), 772-785.

Zhao, Q. ve Rao, S., 1997. Nonparametric renewal function estimation based on estimated densities. Asia-Pacific Journal of Operational Research, 14 (1), 115-126. 